ARTICLE

\title{
Structural mechanism for gating of a eukaryotic mechanosensitive channel of small conductance
}

\author{
Zengqin Deng (10 1,2, Grigory Maksaev (10 1,2,8, Angela M. Schlegel (1) 3,4,8, Jingying Zhang ${ }^{1,2}$, Michael Rau (1) ${ }^{5}$, \\ James A. J. Fitzpatrick (1D 1,5,6,7, Elizabeth S. Haswell (1D ${ }^{3,4}$ \& Peng Yuan (1D) $1,2 \times$
}

Mechanosensitive ion channels transduce physical force into electrochemical signaling that underlies an array of fundamental physiological processes, including hearing, touch, proprioception, osmoregulation, and morphogenesis. The mechanosensitive channels of small conductance (MscS) constitute a remarkably diverse superfamily of channels critical for management of osmotic pressure. Here, we present cryo-electron microscopy structures of a MscS homolog from Arabidopsis thaliana, MSL1, presumably in both the closed and open states. The heptameric MSL1 channel contains an unusual bowl-shaped transmembrane region, which is reminiscent of the evolutionarily and architecturally unrelated mechanosensitive Piezo channels. Upon channel opening, the curved transmembrane domain of MSL1 flattens and expands. Our structures, in combination with functional analyses, delineate a structural mechanism by which mechanosensitive channels open under increased membrane tension. Further, the shared structural feature between unrelated channels suggests the possibility of a unified mechanical gating mechanism stemming from membrane deformation induced by a non-planar transmembrane domain.

\footnotetext{
${ }^{1}$ Department of Cell Biology and Physiology, Washington University School of Medicine, Saint Louis, MO 63110, USA. ${ }^{2}$ Center for the Investigation of Membrane Excitability Diseases, Washington University School of Medicine, Saint Louis, MO 63110, USA. ${ }^{3}$ Department of Biology, Washington University in Saint Louis, Saint Louis, MO 63130, USA. ${ }^{4}$ NSF Center for Engineering Mechanobiology, Washington University in Saint Louis, Saint Louis, MO 63130, USA. ${ }^{5}$ Washington University Center for Cellular Imaging, Washington University School of Medicine, Saint Louis, MO 63110, USA. ${ }^{6}$ Department of Neuroscience, Washington University School of Medicine, Saint Louis, MO 63110, USA. ${ }^{7}$ Department of Biomedical Engineering, Washington University in Saint Louis, Saint Louis, MO 63130, USA. ${ }^{8}$ These authors contributed equally: Grigory Maksaev, Angela M. Schlegel. ${ }^{凶}$ email: yuanp@wustl.edu
} 
$\mathrm{M}$ echanical force sensation mediated by mechanosensitive (MS) ion channels represents a prevalent and fundamental biological process essential for all kingdoms of life ${ }^{1-3}$. MS channels underlie osmoregulation in bacteria, hearing, touch, and proprioception in animals, and are proposed to underlie response to osmotic stress, touch, vibration, and gravity and developmental signals in plants ${ }^{4-11}$. The prokaryotic MS channel of small conductance (MscS) opens in response to hypoosmotic downshock, allowing rapid efflux of solvent and solutes and thereby protecting bacterial cells from rupture ${ }^{12-14}$. Found in many organisms including bacteria, fungi, algae, and plants, MscS-Like (MSL) channels form a remarkably diverse superfamily of MS channels that appear to be pivotal for management of osmotic pressure ${ }^{3}$.

Intrinsically sensitive to membrane tension, MscS channels from several prokaryotes have been extensively characterized and served as a prevailing model system for understanding physicochemical principles in mechanotransduction ${ }^{9}$. X-ray and cryo-electron microscopy (Cryo-EM) structures reveal that each protomer of the homo-heptameric MscS channel consists of three transmembrane helices (TM1-3) followed by a cytoplasmic barrel structure ${ }^{15-22}$. The presumed closed and open structures suggest that TM1 and TM2 constitute a peripheral membrane 'tension sensor', which is attached to the central pore-lining helix TM3a followed by the amphipathic TM3b running approximately parallel to the membrane ${ }^{15-18}$. According to this model, rotation and tilting of TM1 and TM2 as a rigid body under elevated membrane tension, accompanied by displacement of channel-bound lipid molecules, pulls TM3a to open the hydrophobic pore gate ${ }^{16,18}$. However, different gating models derived from electron paramagnetic resonance (EPR) spectroscopy, molecular dynamics (MD) simulations, and recent cryo-EM studies have also been proposed ${ }^{20,23-26}$, and these competing models are actively debated ${ }^{9,20}$.

Much less is known about the structure of eukaryotic members of the MscS superfamily. With only limited homology in the pore-lining helix and the subsequent cytosolic portion, many acquire extra transmembrane helices as well as additional extramembrane domains ${ }^{3}$. These added structural elements may give rise to rich channel properties ${ }^{27}$, which have probably evolved to fulfill adapted functionalities in particular physiological settings. Fundamental questions naturally arise. How are these extra transmembrane helices organized in the membrane? Do they sense mechanical stimulation and contribute to channel gating?

Ten MSL channels have been identified in the land plant $A$. thaliana, and they exhibit distinct membrane topology, domain organization, cellular localization, and physiological functions ${ }^{3}$. MSL1, predicted to contain five transmembrane helices and localized to the inner membranes of mitochondria, is involved in the regulation of membrane potential and maintenance of redox homeostasis under abiotic stress ${ }^{28}$. When heterologously expressed in giant $E$. coli spheroplasts, MSL1 displays stretch-activated gating in excised patches and channel properties similar to those of $E$. coli MscS $(E c M s c S)^{28}$, which demonstrates that MSL1 forms a functional mechanosensitive channel. To gain insights into how mechanosensitive channels gate, we have determined structures of A. thaliana MSL1 (AtMSL1), presumably representing the closed and open conformations, by single-particle cryo-EM. Our structures, in combination with electrophysiology experiments, reveal a structural mechanism by which (at least some) mechanosensitive channels open in response to increased membrane tension.

\section{Results}

Structure determination of the AtMSL1 channel. We expressed an AtMSL1 construct consisting of amino acids 80-497, which lacks the mitochondrial targeting sequence, in the yeast
$P$. pastoris and determined the cryo-EM structure at an overall resolution of $\sim 3.1 \AA$ (Fig. 1a-c, Supplementary Figs. 1, 2, Table 1). The overall architecture and domain organization of the heptameric AtMSL1 channel resemble those of the EcMscS channel (Supplementary Figs. 3 and 4). In contrast to EcMscS, which comprises three membrane-spanning helices (TM1-3), AtMSL1 indeed possesses two additional $\mathrm{N}$-terminal transmembrane helices, resulting in a transmembrane domain with five membrane-spanning helices (TM1-5). Akin to TM3 in EcMscS, the innermost helix TM5 is kinked such that TM5a lines the pore and TM5b runs tangentially to the central pore axis and connects to the middle and C-terminal domains, which are located in the mitochondrial matrix (Fig. 1c). The transmembrane domain is assembled as a seven-bladed propeller, in which each blade comprises peripheral transmembrane helices (TM1-4) organized approximately in a straight line surrounding the pore-lining helix TM5. This unusual structural arrangement creates large unoccupied spaces between the blades, which would presumably be filled with lipids in a membrane environment (Fig. 1b). Probably owing to limited protein-protein contacts between blades, the peripheral transmembrane helices TM1-4 are less well resolved than the remaining part of the channel in the cryo-EM density map (Fig. 1a, Supplementary Fig. 2). Density for the first Nterminal transmembrane helix TM1, which is located at the outer perimeter, is present in the cryo-EM map but rather weak, and thus TM1 is not modeled in the structure (Fig. 1c). The middle and C-terminal domains of AtMSL1, including the extramembrane side portals, are analogous to those of EcMscS except that AtMSL1 lacks the C-terminal seven-stranded $\beta$-barrel present at the extreme C-terminus of EcMscS (Supplementary Fig. 4).

Our structure is further corroborated by a recent independent cryo-EM structure of AtMSL1 that was expressed in mammalian cells $^{29}$. The root-mean-square deviation (r.m.s.d) between these two structures is $\sim 1.4 \AA$ for $\mathrm{Ca}$ atoms from TM4 to the Cterminus. The most pronounced differences arise from the peripheral TM2 and TM3 helices that appear to be dynamic owing to lack of protein-protein interactions within the heptameric channel (r.m.s.d $\sim 3.1 \AA$ for $\mathrm{Ca}$ atoms in TM2 and TM3).

Bowl-shaped transmembrane domain. The bowl-shaped transmembrane domain of AtMSL1 suggests that it naturally resides in locally curved, rather than planar, biological membranes (Fig. 2a). This notion is further supported by the distribution of charged residues in these transmembrane helices, which presumably demarcate the boundary of lipid bilayers (Fig. 2a). The cryo-EM structure was determined in detergent micelles that are virtually devoid of lipids. Thus, to assess whether this unusual shape is an intrinsic property of AtMSL1, rather than an artifact owing to the detergent environment, we also determined the cryo-EM structure of AtMSL1 embedded into lipid nanodiscs, which closely mimic biological membranes ${ }^{30}$, at a lower resolution of $3.4 \AA$ (Supplementary Fig. 5). AtMSL1 structures in nanodiscs and detergents are essentially identical (r.m.s.d of $\sim 0.1 \AA$ for all $\mathrm{Ca}$ atoms), demonstrating that the curved transmembrane region is inherent to AtMSL1. In line with our structures, a low-resolution $(\sim 13 \AA)$ cryo-EM map of the bacterial MscS homolog YnaI, which also contains five TM helices, hinted at a curved membrane surrounding its tapered transmembrane domain ${ }^{31}$. Furthermore, our AtMSL1 structures are reminiscent of the eukaryotic mechanosensitive Piezo channels, which contain extended arms that are apparently curved within a membrane $e^{32-34}$. This shared structural feature between evolutionarily and architecturally unrelated MS channels points to the possibility of a unified 

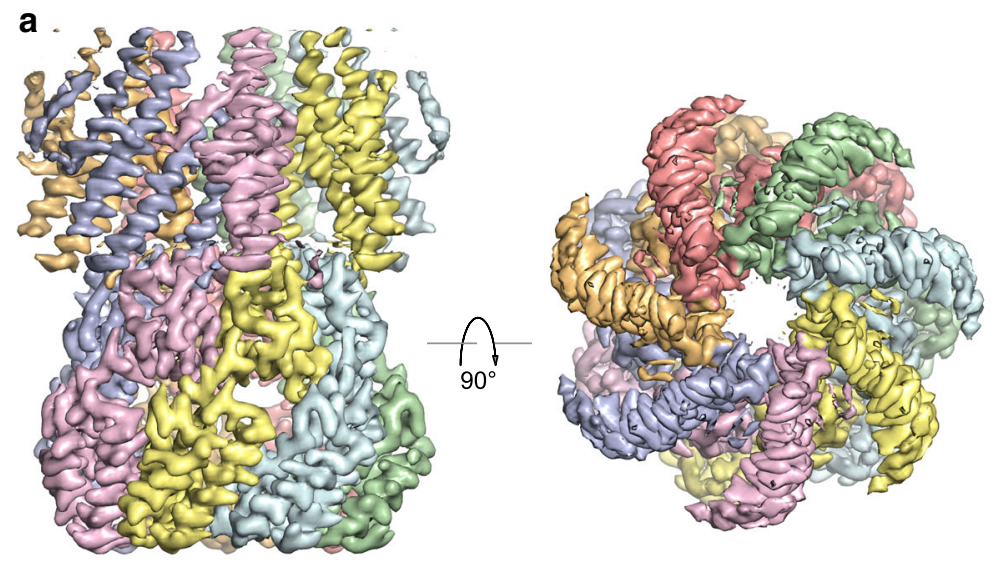

C $\quad \mathrm{N}$

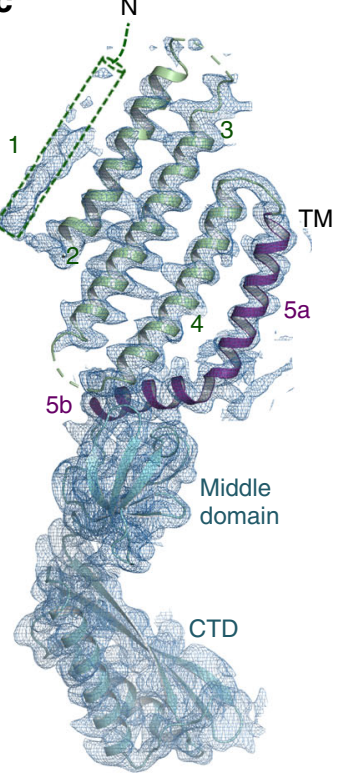

b
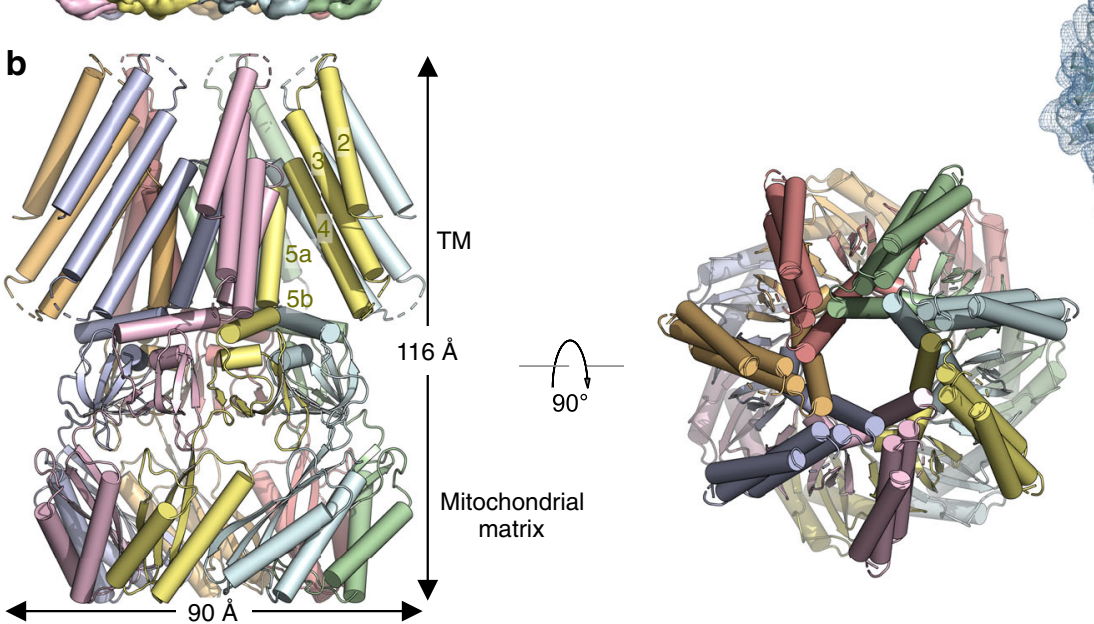

Fig. 1 Cryo-EM structure of AtMSL1. a Orthogonal views of the cryo-EM density contoured at $7.0 \sigma$. Each subunit is uniquely colored. $\mathbf{b}$ Orthogonal views of the MSL1 structure. Transmembrane helices TM2-5, the C-terminal extramembrane domain located in the mitochondrial matrix, and the dimension of the channel are indicated. c A single subunit of MSL1 is shown in cartoon representation with TM helices (TM2-4 in green and TM5 in purple) and extramembrane domains (cyan) indicated. Cryo-EM density contoured at $6.5 \sigma$ is shown in blue mesh.

mechanical gating mechanism stemming from membrane deformation induced by a non-planar transmembrane domain ${ }^{32}$.

In $E c \mathrm{MscS}$, two rings of bulky hydrophobic side chains from L105 and L109 create a 'vapor lock' that blocks ion passage by dewetting the channel pore ${ }^{35}$. The corresponding residues in AtMSL1, V319 and F323, likely play an equivalent role in forming a hydrophobic gate (Fig. 2b, c). Pore radius calculation reveals that F323, near the C-terminal end of the pore-lining helix TM5a, defines the narrowest constriction along the central permeation pathway, which is comparable to the closed $E c M s c S$ gate in dimension. This indicates that the AtMSL1 structure represents a non-conducting, resting state, which is consistent with the absence of membrane tension in detergent micelles and lipid nanodiscs where the structures were determined.

Open conformation of AtMSL1. Like TM3a in $E c M s c S^{24,36}$, the pore-lining helix TM5a in AtMSL1 contains several glycine and alanine residues with small side chains, which give rise to tight helix-helix interfaces in the closed conformation (Supplementary Fig. 3, Fig. 3a, b). Perturbation of this critical interface by the introduction of a bulkier side chain at position 106 in $E c \mathrm{MscS}$ (A106V) resulted in an X-ray structure in a presumed open conformation ${ }^{16}$. This position, strategically located between two consecutive pore narrowing residues (L105 and L109), is highly conserved among MscS homologs and appears to be essential for the tight helix packing that stabilizes the closed conformation (Fig. 3a, b). Inspired by these observations, we introduced the equivalent mutation in AtMSL1 (A320V) to evaluate whether analogous destabilization accordingly favors an open conformation.

Electrophysiological analyses of EcMscS, AtMSL1, and AtMSL1 A320V expressed in giant E. coli spheroplasts indicated that AtMSL1 A320V exhibits much longer open dwell times (that is, the amount of time spent in the open or partially open states) upon tension release than AtMSL1 when gating is triggered by a brief application of negative pressure (Fig. 3c). Once gated, 90\% of AtMSL1 gating events had open dwell times of less than $20 \mathrm{~s}$, compared to $44 \%$ of AtMSL1 A320V gating events (Fig. 3d). Only $2 \%$ of AtMSL1 gating events had open dwell times above $80 \mathrm{~s}$, compared to $50 \%$ of AtMSL1 A320V gating events.

Both AtMSL1 and AtMSL1 A320V exhibited subconducting states, but this was more frequently observed with AtMSL1 A320V (Table 2, Fig. 3c). Furthermore, AtMSL1 A320V was more likely than AtMSL1 to open immediately after patch formation and prior to the application of pressure ramps as the patch of membrane within the glass pipette has inherent lateral tension due to the lipid-glass adhesion force (Table 2). EcMscS, AtMSL1, and AtMSL1 A320V had statistically indistinguishable unitary conductances and relative gating pressure thresholds $(0.49 \pm 0.16$, $0.64 \pm 0.14$, and $0.55 \pm 0.15$, respectively) when measured at a membrane potential of $-70 \mathrm{mV}$ (Table 3). However, unlike $E c M s c S^{14,26}$, neither AtMSL1 nor AtMSL1 A320V displayed inactivation in response to repeated pressure ramps, regardless of ramp rate or length. Thus, the A320V mutation is likely to stabilize the open state of AtMSL1 or to kinetically impede its closure. 
a

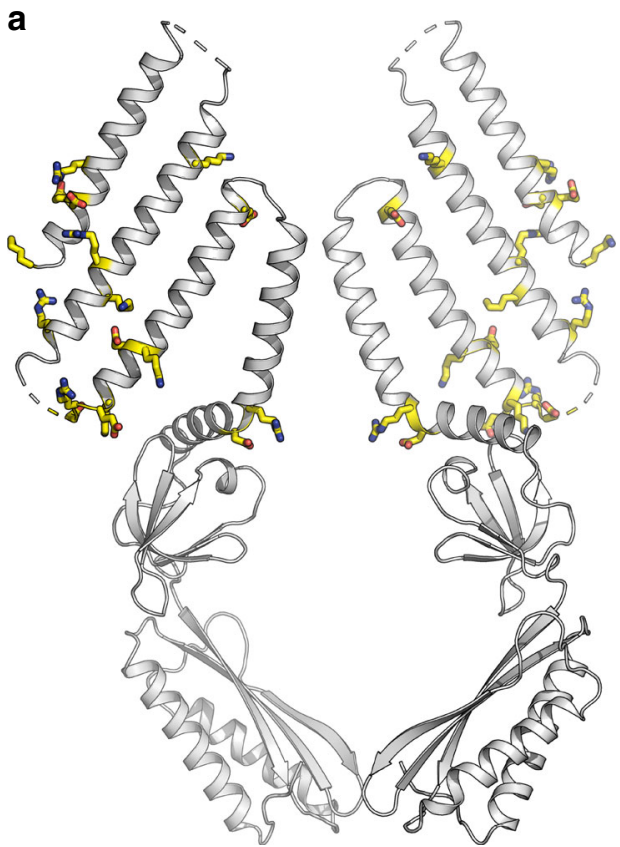

b

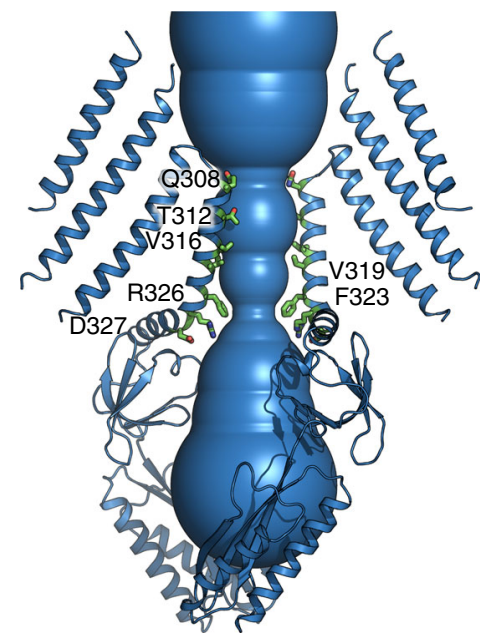

C

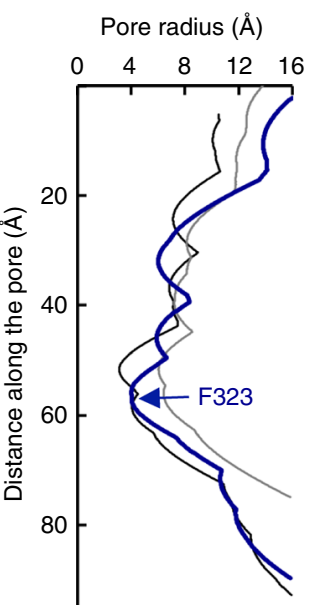

Fig. 2 The closed conformation of AtMSL1. a Distribution of charged residues, shown in stick representation, in the transmembrane helices indicates a curved transmembrane region. Only two opposing MSL1 subunits are shown for clarity. $\mathbf{b}$ The pore structure. Residues lining the pore are highlighted in stick representation. c The pore profile of MSL1 (blue). Also shown are the pore profiles of the closed (black, PDB: 6RLD) and open (gray, PDB: 2VV5) EcMscS structures.

\section{Table 1 Cryo-EM data collection, refinement and validation statistics.}

\begin{tabular}{|c|c|c|c|}
\hline & $\begin{array}{l}\text { AtMSL1 in detergents (EMDB: 21444) } \\
\text { (PDB: 6VXM) }\end{array}$ & $\begin{array}{l}\text { AtMSL1 A320V in detergents (EMDB: } \\
\text { 21445) (PDB: 6VXN) }\end{array}$ & $\begin{array}{l}\text { AtMSL1 in nanodiscs (EMDB: 21447) } \\
\text { (PDB: } 6 \text { VXP) }\end{array}$ \\
\hline Magnification & 105,000 & 105,000 & 105,000 \\
\hline Voltage (kV) & 300 & 300 & 300 \\
\hline Defocus range $(\mu \mathrm{m})$ & -1.0 to -2.5 & -1.0 to -2.5 & -1.0 to -2.5 \\
\hline Pixel size $(\AA)$ & 1.1 & 1.1 & \\
\hline Symmetry imposed & C7 & C7 & C7 \\
\hline Initial particle images (no.) & 936,130 & 428,889 & 457,683 \\
\hline FSC threshold & 0.143 & 0.143 & 0.143 \\
\hline $\begin{array}{l}\text { Map resolution range }(\AA) \\
\text { Refinement }\end{array}$ & $2.5-6.0$ & $2.5-6.0$ & $3.0-7.0$ \\
\hline Initial model used (PDB code) & $5 \mathrm{AJI}$ & This study & This study \\
\hline Model resolution $(\AA)$ & 3.0 & 3.1 & 3.5 \\
\hline FSC threshold & 0.5 & 0.5 & 0.5 \\
\hline Model resolution range $(\AA)$ & 2.7 & 2.7 & 3.1 \\
\hline Protein & 36.2 & 93.1 & 73.6 \\
\hline Ligand & 20 & 111 & $\mathrm{~N} / \mathrm{A}$ \\
\hline \multicolumn{4}{|l|}{ R.m.s. deviations } \\
\hline Bond lengths $(\AA)$ & 0.006 & 0.008 & 0.008 \\
\hline Bond angles $\left({ }^{\circ}\right)$ & 0.763 & 1.283 & 1.126 \\
\hline \multicolumn{4}{|l|}{ Validation } \\
\hline MolProbity score & 1.57 & 1.28 & 1.64 \\
\hline Clashscore & 5.58 & 5.31 & 6.59 \\
\hline Poor rotamers (\%) & 0 & 0 & 0 \\
\hline \multicolumn{4}{|l|}{ Ramachandran plot } \\
\hline Favored (\%) & 96.1 & 98.45 & 95.94 \\
\hline Allowed (\%) & 3.9 & 1.55 & 4.06 \\
\hline Disallowed (\%) & 0 & 0 & 0 \\
\hline
\end{tabular}


a

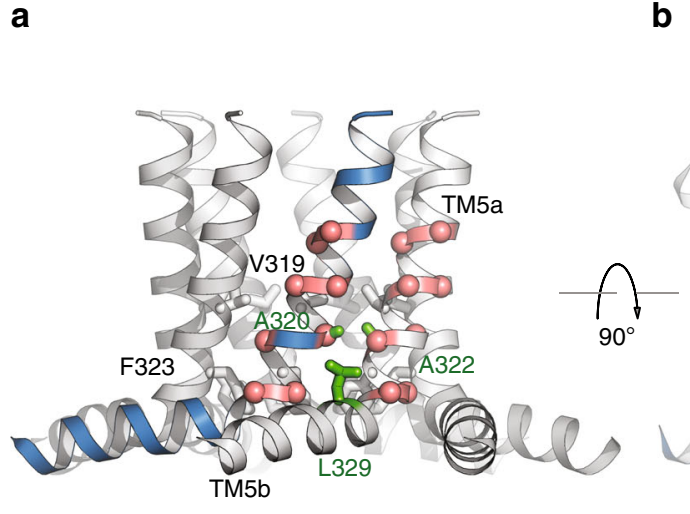

b

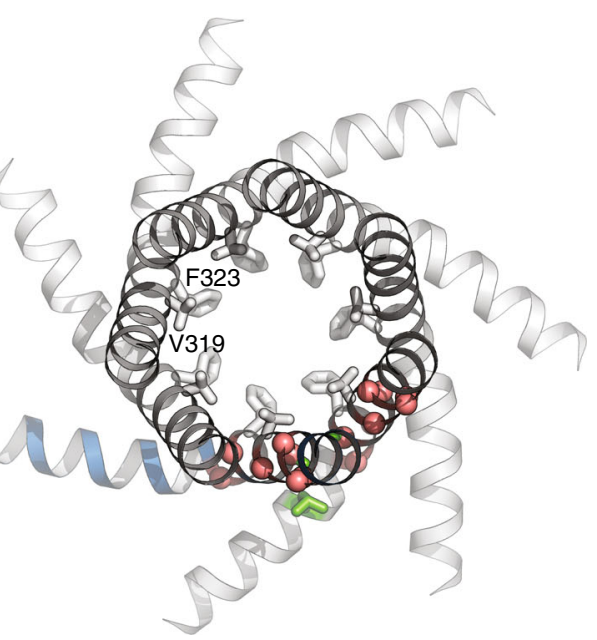

C
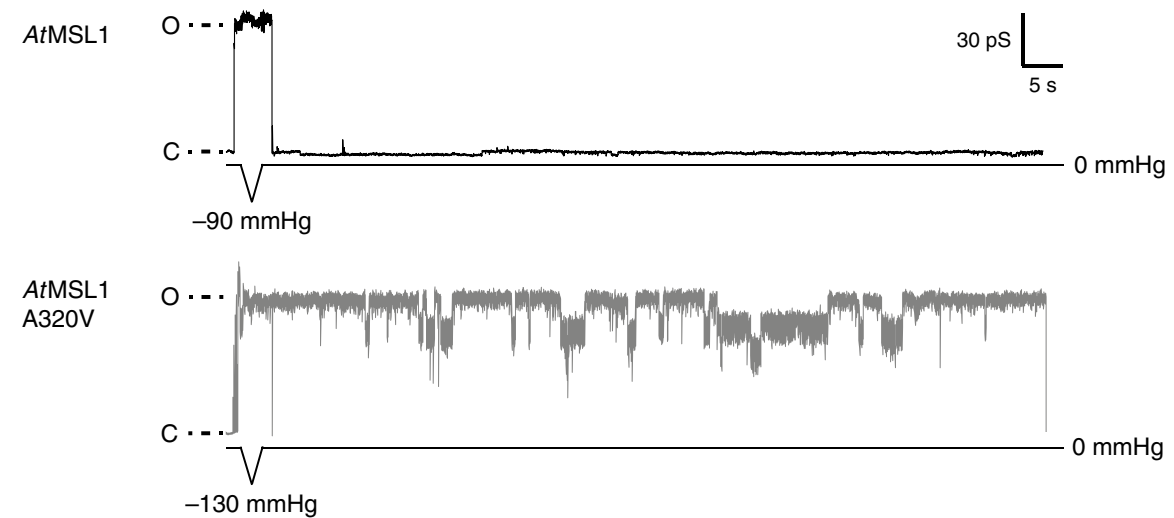

d

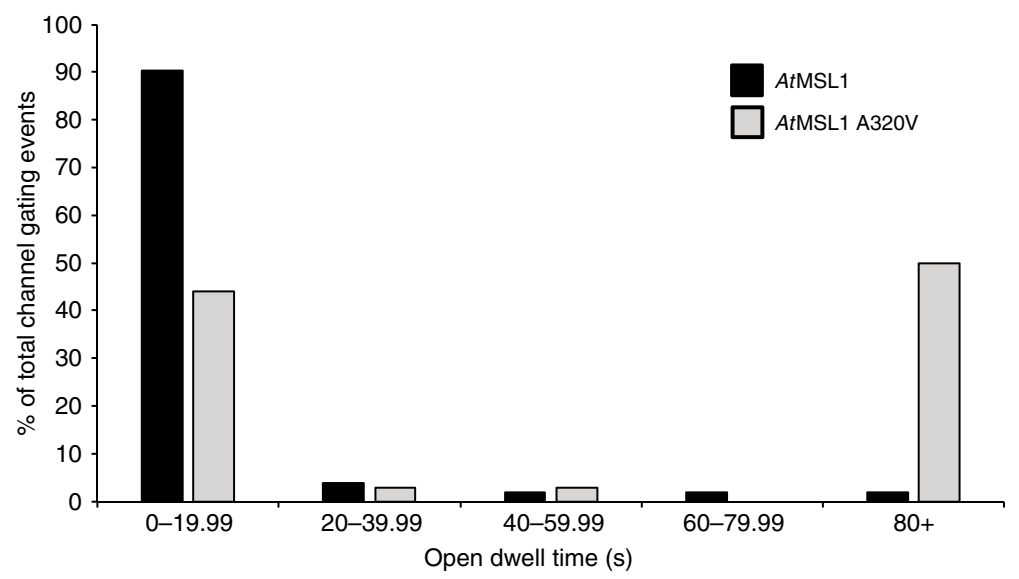

Fig. 3 Helix-helix interface and the A320V mutant. a, b Orthogonal views of TM5. Glycine and alanine residues are indicated as red spheres. A320 and its contacts in a neighboring helix (A322 and L329) are highlighted in green. V319 and F323 constitute the hydrophobic gate. c Representative traces for single AtMSL1 and A320V channel activities in response to a brief $1 \mathrm{~s}$ pressure ramp followed by $90 \mathrm{~s}$ without pressure. Closed state (baseline) current is indicated by $\mathrm{C}$ while open states are indicated by $\mathrm{O}$. The maximum negative pressure used is indicated. Membrane potential was clamped at $-70 \mathrm{mV}$. Multiple subconducting states were observed with the A320V mutant, while the peak unitary current of A320V was indistinguishable from that of the wild type (Table 3). d Percentages of AtMSL1 (black) and AtMSL1 A320V (gray) gating events with open dwell times of 0-19.99 s, 20-39.99s, 40-59.99 s, $60-79.99 \mathrm{~s}$, or $80+\mathrm{s}$. Data represent traces obtained from 10 patches per variant channel with only the first gating event analyzed per trace (103 traces for AtMSL1, 34 for AtMSL1 A320V). Source data are provided as a Source Data file.

We determined the cryo-EM structure of the AtMSL1 A320V mutant in detergents to an overall resolution of $\sim 3.0 \AA$ (Fig. $4 \mathrm{a}, \mathrm{b}$, Supplementary Fig. 6, Table 1). Side views of the reference-free $2 \mathrm{D}$ projections indicate marked changes in the transmembrane region of A320V compared with that of the wild-type AtMSL1 (Supplementary Fig. 7a-c). The bowl-shaped transmembrane region observed in the wild-type channel flattens, which is accompanied by considerable in-plane expansion. Flattening and expansion were recapitulated in reconstitution of A320V into lipid nanodiscs (Supplementary Fig. 7d), though A320Vnanodiscs did not yield high-resolution 3D reconstruction. In the structure of $\mathrm{A} 320 \mathrm{~V}$ in detergents, the diameter of the 
Table 2 Spontaneous opening and subconducting states for AtMSL1 and A320V.

\begin{tabular}{lll|} 
& AtMSL1 & AtMSL1 A320V \\
\hline $\begin{array}{l}\text { Channel(s) open before } \\
\text { pressure ramp }\end{array}$ & $\begin{array}{l}25 \%(3 / 12 \\
\text { traces) }\end{array}$ & $57 \%$ (8/14 traces) \\
$\begin{array}{l}\text { Channel exhibits } \\
\text { subconducting state }\end{array}$ & $\begin{array}{l}29 \%(30 / 102 \\
\text { traces) }\end{array}$ & $50 \%$ (17/34 traces) \\
$\begin{array}{l}\text { Subconducting state follows full } \\
\text { opening }\end{array}$ & $\begin{array}{l}17 \%(5 / 30 \\
\text { traces) }\end{array}$ & $82 \%$ (14/17 traces) \\
\hline
\end{tabular}

Table 3 EcMscS and AtMSL1 and A320V have similar
conductances and relative gating pressures.

\begin{tabular}{llcl} 
& EcMscS & AtMSL1 & AtMSL1 A320V \\
\hline $\begin{array}{l}\text { Conductance }(\mathrm{nS}) \\
\text { Relative gating }\end{array}$ & $1.02 \pm 0.17$ & $1.12 \pm 0.16$ & $0.98 \pm 0.12$ \\
pressure $\left(P_{\mathrm{x}} / P_{\mathrm{MscL}}\right)$ & $0.49 \pm 0.16$ & $0.63 \pm 0.14$ & $0.55 \pm 0.15$ \\
\hline $\begin{array}{l}\text { Channel activities were triggered by application of } 2.5 \mathrm{~s} \text { symmetric pressure ramps at } \\
\text { membrane potentials of }-70 \mathrm{mV} \text {. Values are averages } \pm \text { standard deviation. No difference in } \\
\text { conductance or gating pressure relative to endogenous } E \text { EMscL was observed between wild- } \\
\text { type } A \text { AtMSL1 and A320V using one-way ANOVA followed by post hoc Tukey's test, } p<0.05 . \\
N=10 \text { patches per variant. Source data are provided as a Source Data file. }\end{array}$ \\
\hline
\end{tabular}

transmembrane domain is increased from 90 to $105 \AA$ while the height of the channel is reduced from 116 to $92 \AA$, compared with those in the wild-type structure (Fig. $4 \mathrm{a}, \mathrm{b}$ ). These drastic global conformational changes in the transmembrane domain result in substantial opening of the central pore, which increases in diameter from $\sim 8 \AA$ in the wild type to $\sim 20 \AA$ in A320V (Fig. $4 \mathrm{c}$, d). Therefore, we conclude that the A320V structure represents an open, conducting conformation.

Gating mechanism. On the basis of these two structures, we can infer that the transition from the closed to the open state of MSL1 involves pronounced structural rearrangements in the transmembrane domain, whereas the soluble domains in the mitochondrial matrix remain essentially unchanged (Fig. 4e, f, Supplementary Movie 1) (r.m.s.d of $\sim 1.1 \AA$ for $\mathrm{Ca}$ atoms of residues 341-491). Upon channel opening, the peripheral helices TM2-4 approximately move as a rigid body by a rotation of $\sim 135^{\circ}$ (approximately about the helical axis of TM3) and a translation of $\sim 30 \AA$. These conformational changes give rise to a flattened and expanded transmembrane domain in a lipid bilayer, in which transmembrane helices are more densely associated (Fig. $4 \mathrm{~g}, \mathrm{~h}$ ). The pore-lining helix TM5a becomes more tilted within the membrane and joins TM5b without an apparent kink (Fig. 4e, f). Concomitantly, the outward movement of the straightened TM5 helix results in wide opening of the hydrophobic gate (Fig. $4 \mathrm{~h}$, Supplementary Fig. 8), which renders the conduction path less hydrophobic at this region (Supplementary Fig. 9). The increased pore diameter and decreased hydrophobicity support our conclusion that the A320V structure represents an open, conductive conformation. Consistently, straightening of the pore-lining helix was suggested in a simulated open EcMscS conformation, which was further supported by patch-clamp experiments of alanine substitutions at positions near the $\mathrm{kink}^{24}$. In the open structure of AtMSL1 A320V, fewer lipid-attributable densities are observed between transmembrane helices than in the closed state (Supplementary Fig. 8), which is in accordance with the proposal that

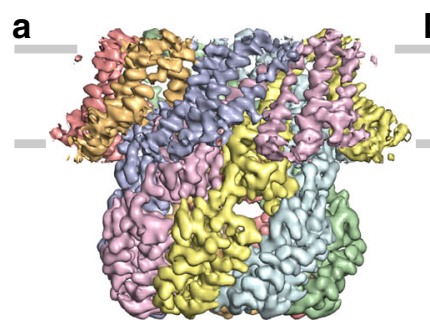

b

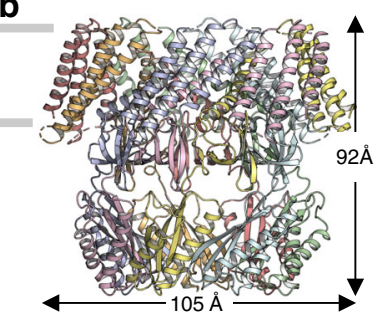

C

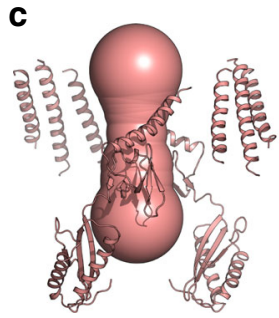

d Pore radius $(\AA)$

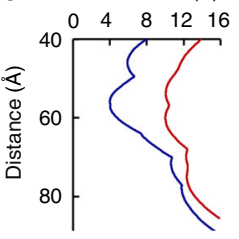

e

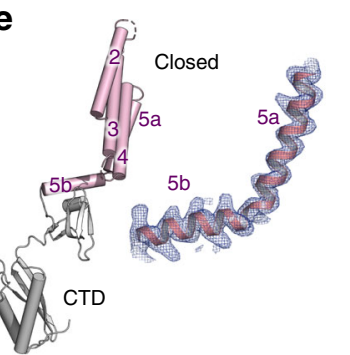

$\mathbf{f}$
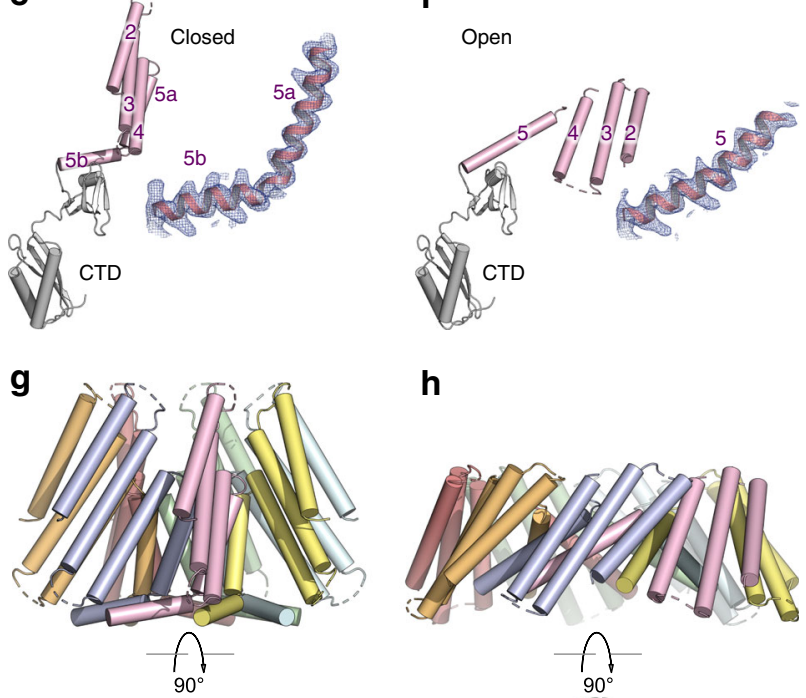

h
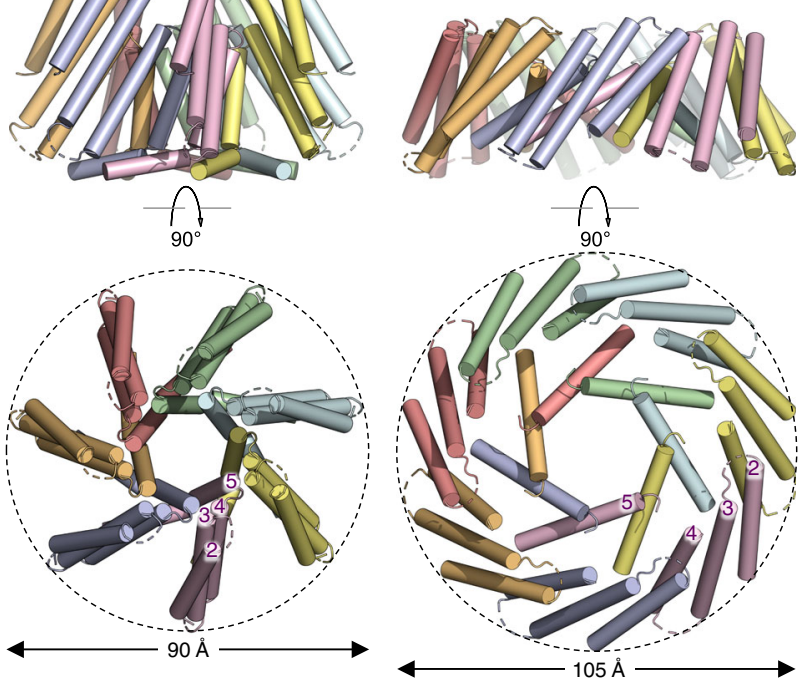

Fig. 4 Structure of $\mathbf{A} 320 \mathrm{~V}$ and channel opening. a Cryo-EM density, contoured at $5.0 \sigma$, for the A320V mutant. Each subunit is in a unique color. b Structure of A320V. The dimension of the channel is indicated. c The wide-open pore of A320V. d The pore profiles of the closed wild type (blue) and the open A320V mutant (red). e, f Structures of a single subunit for the wild type (e) and the A320V mutant channels (f). The Cterminal extramembrane domain in the mitochondrial matrix is colored in gray and the transmembrane region is colored in magenta. Cryo-EM densities, contoured at $7.0 \sigma$, are shown for TM5. $\mathbf{g}$, h Orthogonal views of the transmembrane domains of the wild-type MSL1 (g) and the A320V mutant (h). 
channel-bound lipids repartition to the bulk membrane bilayer under increased membrane tension to promote channel opening in $\mathrm{EcMscS}^{18}$.

Thus, a single mutation in the pore-lining helix facilitates channel opening in both prokaryotic and eukaryotic MscS channels. However, conformational changes involved in channel gating show important similarities and differences between EcMscS and AtMSL1 (Supplementary Movies 1, 2; Supplementary Fig. 9). While the soluble domains remain virtually stationary during gating, the transmembrane domains experience substantial rearrangements. Both $E c \mathrm{MscS}$ and AtMSL1 channels appear to possess a peripheral 'tension sensor' (TM1-2 in EcMscS and TM1-4 in AtMSL1) that is attached to the pore-lining helix and undergoes dramatic repositioning upon activation. However, the direction and nature of the 'tension sensor' movements of the two channels are in marked contrast. Ultimately, the pore-lining helix becomes more perpendicular to the membrane plane in $E c \mathrm{MscS}^{16,18}$, but more tilted in AtMSL1 upon channel opening.

\section{Discussion}

For a channel embedded in a membrane with lateral tension $(\sigma)$, the free energy difference between the closed and open conformations can be expressed as

$$
\Delta G=\left(\Delta G_{\text {channel }}+\Delta G_{\text {bending }}\right)-\sigma \Delta A,
$$

where $\Delta G_{\text {channel }}$ is the difference of free energy intrinsic to channel gating, $\Delta G_{\text {bending }}$ the free energy difference of membrane bending associated with channel gating, and $\Delta A$ the difference of in-plane cross-sectional area of the channel ${ }^{32,37}$. Here, the closed and open structures of AtMSL1 provide a straightforward structural mechanism for gating by lateral membrane tension. Under low tension, the transmembrane region of AtMSL1 is curved. Increased lateral tension favors a more planar bilayer, which drives flattening of the transmembrane 'tension sensor', resulting in an in-plane area expansion of the transmembrane region $\left(\sim 23 \mathrm{~nm}^{2}\right.$ for resolved TM2-TM5 helices) as well as straightening and outward movement of the pore-lining helices that ultimately open the hydrophobic gate. This simple but elegant concept of membrane flattening and area expansion has been previously suggested for mechanical gating of the architecturally unrelated Piezol channel on the basis of its intrinsically curved transmembrane region ${ }^{32}$. Indeed, force-induced flattening and expansion of Piezol have been indicated by atomic force microscopy studies ${ }^{38}$, but no open structure for Piezo channels has been determined. In this work we provide a near-atomic visualization of the membrane flattening and expansion process involved in channel gating. These results may be applicable to MscS homologs harboring even more transmembrane helices, and additional helices could function as an extension of the 'tension sensor' that undergoes force-induced flattening and expansion. Therefore, the structural transition illuminated in this study may represent a unified gating mechanism that underlies numerous mechanotransduction events in all kingdoms of life.

\section{Methods}

Cloning and expression and purification. DNA encoding Arabidopsis thaliana MSL1 (AtMSL1, NCBI: NP_567165.2) was synthesized (Gene Universal Inc.) and served as the template for subsequent cloning. The $\mathrm{N}$-terminal 79 amino acids corresponding to the mitochondrial targeting sequence was removed from the expression construct. Primers 5'-TAGCCTCGAGCCACCATGAGCAGCAAA AGCGATG- $3^{\prime}$ and 5'-CCTTGAAACAAAACTTCCAAAGAATTCGA-3' were used to generate the DNA fragment encoding residues 80-497 of AtMSL1, which was ligated into a modified yeast Pichia pastoris expression vector pPICZ-B that contains a C-terminal PreScission protease cleavage site and a GFP-His ${ }_{10}$ tag. The A320V mutation was generated by site-directed mutagenesis using the primers $5^{\prime}$ GTTACCGCCTTTGCCGCCCGTG- ${ }^{\prime}$ and $5^{\prime}$ - GACGCCACCGACACCACCG- $3^{\prime}$.

For expression in E. coli spheroplasts, AtMSL1 residues 80-497, codonoptimized for expression in E. coli, were synthesized (ThermoFisher Scientific).
This fragment was used to replace His-MSL1 in pET300-His-MSL1 ${ }^{28}$ by Gibson cloning. The resulting pET300-AtMSL1 vector was then linearized and a Cterminal GFP tag preceded by an EcoRI restriction site added by Gibson cloning to pET300-AtMSL1 to create pET300-AtMSL1-GFP. To create pET300-MscS-GFP, the $E c M s c S$ sequence was used to replace the AtMSL1 sequence in pET300AtMSL1-GFP using Gibson cloning. The A320V mutation was introduced into pET300-AtMSL1-GFP by site-directed mutagenesis using the primers $5^{\prime}$ - GTTGGT GGCGTTGTGACCGCATTTGC-3' and 5'- GCAAATGCGGTCACAACGCCACC AAC-3', creating pET300-A320V-GFP.

The wild-type AtMSL1 channel and the A320V mutant were expressed in Pichia pastoris (strain SMD1163H, Invitrogen). Cells were harvested and disrupted by milling (Retsch MM400) and resuspended in buffer containing $50 \mathrm{mM}$ Tris-HCl $\mathrm{pH} 8.0,150 \mathrm{mM} \mathrm{NaCl}$, a mixture of protease inhibitors $(2.5 \mu \mathrm{g} \mathrm{ml}-1$ leupeptin, $1 \mu \mathrm{g} \mathrm{ml}^{-1}$ pepstatin A, $100 \mu \mathrm{g} \mathrm{ml}^{-1}$ 4-(2-Aminoethyl) benzenesulfonyl fluoride hydrochloride, $3 \mu \mathrm{g} \mathrm{ml}^{-1}$ aprotinin, $1 \mathrm{mM}$ benzamidine and $200 \mu \mathrm{M}$ phenylmethane sulphonylfluoride) and DNase I. Cell membranes were solubilized by adding Lauryl Maltose Neopentyl Glycol (LMNG, Anatrace) to a final concentration of $\sim 1 \%$ (w:v) while stirring for $2 \mathrm{~h}$ at $4{ }^{\circ} \mathrm{C}$. Solubilized protein was separated from the insoluble fraction by centrifugation for $0.5 \mathrm{~h}$ at $30,000 \times g$. The suspension was incubated with $3 \mathrm{ml}$ of cobalt-charged resin (G-Biosciences) for $3 \mathrm{~h}$ at $4{ }^{\circ} \mathrm{C}$ with rotation. Resin was then washed with $30 \mathrm{ml}$ buffer containing $20 \mathrm{mM}$ Tris- $\mathrm{HCl} \mathrm{pH} \mathrm{8.0,150} \mathrm{mM} \mathrm{NaCl,} 20 \mathrm{mM}$ imidazole, and $85 \mu \mathrm{M}$ glyco-diosgenin (GDN, Anatrace). The GFP-His ${ }_{10}$ tag was removed by PreScission protease at $4{ }^{\circ} \mathrm{C}$ overnight with gentle rocking. The flow-through containing the channel protein was then collected, concentrated, and further purified on a Superose 6 increase 10/ 300 GL column (GE Healthcare Life Sciences) in $20 \mathrm{mM}$ Tris-HCl pH 8.0, $150 \mathrm{mM}$ $\mathrm{NaCl}$ and $40 \mu \mathrm{M}$ GDN. The peak fractions were combined and concentrated for cryo-EM experiments.

Nanodisc reconstitution. Soybean polar lipid extract (Avanti Polar Lipids, Inc.) in chloroform was dried to form a thin film in a glass tube under argon and then by vacuum desiccation for over $2 \mathrm{~h}$. The lipid film was rehydrated to $10 \mathrm{mM}$ in buffer containing $20 \mathrm{mM}$ Tris- $\mathrm{HCl} \mathrm{pH} \mathrm{8.0,150} \mathrm{mM} \mathrm{NaCl}$, and $14 \mathrm{mM} \mathrm{DDM}$, and sonicated immediately before use. Protein with C-terminal GFP-His ${ }_{10}$ tag was eluted from the cobalt-charged resin and concentrated to $\sim 45 \mu \mathrm{M}$ and then mixed with the scaffold protein MSP2N2 and lipids in a final molar ratio of $\sim 1: 0.5: 50$. The mixture was incubated on ice for $10 \mathrm{~min}$ before the addition of Bio-beads SM-2 (Bio-Rad) to a final volume of $\sim 12.5 \%(\mathrm{v} / \mathrm{v})$ to remove the detergent. In the meanwhile, PreScission protease was also added to cleave the C-terminal GFP$\mathrm{His}_{10}$ tag. The resulting mixture was incubated at $4{ }^{\circ} \mathrm{C}$ overnight with constant rotation. The supernatant was isolated by centrifugation, concentrated and further purified on a Superose 6 increase 10/300 GL column (GE Healthcare Life Sciences) in buffer containing $20 \mathrm{mM}$ Tris- $\mathrm{HCl} \mathrm{pH} 8.0$ and $150 \mathrm{mM} \mathrm{NaCl}$.

Cryo-EM sample preparation and imaging. Cryo-EM grids were prepared with FEI Vitrobot Mark IV (FEI). $3.5 \mu \mathrm{l}$ of purified channel protein $\left(\sim 6 \mathrm{mg} \mathrm{ml}^{-1}\right.$ in detergent micelles or $\sim 3 \mathrm{mg} \mathrm{ml}^{-1}$ in lipid nanodiscs) was applied onto glowdischarged copper Quantifoil R2/2 holey carbon grids (Quantifoil). Grids were blotted for $2 \mathrm{~s}$ at $\sim 100 \%$ humidity and flash frozen in liquid ethane. For imaging the grids were loaded into a Titan Krios (FEI) electron microscope operating at $300 \mathrm{kV}$, which is equipped with GIF Quantum energy filter and a Gatan K2 Summit (Gatan) detector. Movies were recorded using the EPU software (https:// www.fei.com/software/epu-automated-single-particles-software-for-life-sciences/) in super-resolution mode with a pixel size of $0.55 \AA$ and a nominal defocus value between -1.0 and $-2.5 \mu \mathrm{m}$. Data were collected with a dose of $\sim 7.8$ electrons per $\AA^{2}$ per second, and each movie was recorded by 40 frames ( $200 \mathrm{~ms}$ per frame) for an $8 \mathrm{~s}$ exposure.

Image processing and map calculation. Image stacks were first aligned, binned by 2 , and dose-weighted using MotionCor $2^{39}$, and then subjected to contrast transfer function (CTF) determination using GCTF ${ }^{40}$. Following motion correction and CTF estimation, low-quality images were manually removed from the datasets. For AtMSL1 in detergent GDN, 1031 particles were manually selected to compute two-dimensional class templates for automated particle picking in RELION $3^{41}$. A total of 936,130 particles were automatically picked from 3618 micrographs. A box size of 256 pixels was used for particle extraction and a mask diameter of $190 \AA$ A was used for 2D classification. Two rounds of 2D classification were performed to identify particles representing the channel (454,555 particles), which were imported into cryoSPARC 22 to generate an initial map for 3D classification in RELION3. Three classes showing intact channel features (158,172 particles) were selected and subjected to 3D refinement, yielding an overall resolution of $3.48 \AA$ A. CTF refinement and Bayesian polishing further improved the resolution to $3.06 \AA$ after masked 3D refinement.

For AtMSL1 in nanodiscs, 2595 particles were manually picked to generate 2D class templates for automated particle picking in RELION3, which resulted in a total of 457,683 particles from 3486 micrographs. After two rounds of $2 \mathrm{D}$ classification, 140,548 particles were selected for 3D classification using low-pass filtered map of AtMSL1 in detergent micelles as the initial model. One class (23,077 particles) was selected for 3D refinement, reaching an overall resolution of $4.2 \AA$. 
CTF refinement and Bayesian polishing improved the resolution to $3.39 \AA$ after masked 3D refinement.

For the AtMSL1 A320V mutant in detergents, 5553 particles were picked using LoG-based auto-picking to generate $2 \mathrm{D}$ classes for automatic picking in RELION3. Automatic picking resulted in 428,889 particles from 1947 micrographs. From two rounds of 2D classification, 192,899 particles were selected and imported into cryoSPARC to calculate an initial map for 3D classification requesting four classes in RELION3. Two classes (96,781 particles) containing clear TM densities were selected for further 3D refinement, reaching an overall resolution of $3.86 \AA$. 3D classification without image alignment was performed and two classes (74,578 particles) were selected for further 3D refinement, yielding a $3.81 \AA$ A reconstruction. CTF refinement and Bayesian polishing improved the resolution to $3.27 \AA$ after 3D refinement. 3D classification without image alignment using the polished particles was performed, and the major class containing 52,883 particles was selected for masked 3D refinement, reaching a final overall resolution to $2.96 \AA$.

For A320V in nanodiscs, 2016 particles were manually selected for 2D class templates. Automatic picking resulted in 464,730 particles from 3234 micrographs. From two rounds of $2 \mathrm{D}$ classification, 65,310 particles were selected. However, 3D reconstruction did not reach resolution beyond $8 \AA$ in cryoSPARC and RELION3, and therefore we did not build the model.

Model building and coordinate refinement. A homology model of the AtMSL1 extramembrane domain was generated using coordinates of $E c M s c S$ (PDB: $5 \mathrm{AJI})$ by the SWISS-MODEL server ${ }^{43}$. The model was placed into the cryo-EM density map by using UCSF Chimera ${ }^{44}$ and then manually rebuilt in COOT ${ }^{45}$. The transmembrane region was de novo built in COOT. Iterative model building in COOT and refinement using real_space_refine in PHENIX ${ }^{46}$ were performed to obtain the final model. The final model shows good geometry and contains amino acids 203-229, 236-269, and 276-491. The structure of AtMSL1 in lipid nanodiscs was built using the AtMSL1 structure in detergent micelles as a reference, followed by multiple rounds of model rebuilding in COOT and refinement using real_space_refine in PHENIX. For the AtMSL1 A320V mutant, resolution of the EM density map is higher in the C-terminal extramembrane domain. Density for the transmembrane region is weaker. The C-terminal domain of the wild-type AtMSL1 was first placed into the EM density map as a rigid body. Owing to weaker density in the transmembrane domain, TM5 was de novo built in COOT, and this partial model comprising the C-terminal domain and TM5 was refined against the map using real_space_refine in PHENIX. In the final steps, TM2-TM4 helices from the wild-type channel were modeled into the A $320 \mathrm{~V}$ cryo-EM density map as a rigid body, and then further refined. The final A320V structure contains residues 204-229, 236-269, 276-302 and 313-491. Final refined models were validated using MolProbity ${ }^{47}$. Pore dimensions were estimated by using HOLE ${ }^{48}$. Structural figures, including the surface hydrophobicity plots ${ }^{49}$, and morphed movies (using the linear interpolation method) were prepared using PyMol (pymol.org) and UCSF Chimera ${ }^{44}$. The phylogenetic tree was generated by MEGA $\mathrm{X}^{50}$.

Electrophysiology. AtMSL1, A320V, and EcMscS were expressed in giant spheroplasts made using E. coli strain MJF516, which lacks $m s c S, m s c K, y b i O$, and $y j e P^{51}$. A lysogenized version of $E$. coli strain MJF516 (generated using the Novagen $\lambda$ DE3 lysogenization kit (Millipore Sigma)) was transformed with pET300-EcMscS-GFP, pET300-AtMSL1-GFP, or pET300-A320V-GFP. Giant spheroplasts were prepared following the protocol ${ }^{52}$ with the following modifications: treatment with cephalexin only was performed for $1 \mathrm{~h}$, at which point isopropyl $\beta$-d-1-

thiogalactopyranoside (IPTG) was added to a final concentration of $1 \mathrm{mM}$ and cultures shaken at $42^{\circ} \mathrm{C}, 180 \mathrm{rpm}$ for an additional hour before being stored overnight at $4{ }^{\circ} \mathrm{C}$ in $50 \mathrm{~mL}$ conical tubes.

Patch-clamp electrophysiology was performed with inside-out excised patches. Pipette buffer contained $200 \mathrm{mM} \mathrm{KCl}, 90 \mathrm{mM} \mathrm{MgCl} 2,5 \mathrm{mM} \mathrm{CaCl}_{2}$, $5 \mathrm{mM}$ Hepes, $\mathrm{pH} 7.4$ and the bath buffer was identical to the pipette buffer with the addition of $400 \mathrm{mM}$ sucrose. Pressure was controlled and applied using an HSPC-1 high speed pressure clamp system (ALA Scientific) and data acquired using an Axon Axopatch 200B amplifier and a Digidata 1440A digitizer (Molecular Devices). All pipettes were of bubble number 4.5 . Data were acquired at $20 \mathrm{kHz}$ for pressure sensitivity and conductance measurements and at $10 \mathrm{kHz}$ for open-state lifetime measurements and all data low-pass filtered at $5 \mathrm{kHz}$. Pressure sensitivity was evaluated at $-70 \mathrm{mV}$ membrane potential using a symmetric pressure ramp consisting of a $2.5 \mathrm{~s}$ increase in applied suction followed by a $2.5 \mathrm{~s}$ suction release. Open state lifetime measurements were performed at a membrane potential of $-70 \mathrm{mV}$ and consisted of a symmetric pressure ramp of $1 \mathrm{~s}$ suction increase followed by $1 \mathrm{~s}$ suction release and continued application of $70 \mathrm{mV}$ pipette potential without additional suction for $95.7 \mathrm{~s}$ after suction release.

Data analysis and measurements were performed using ClampFit 10.6 software (Molecular Devices). For all measurements, traces in which channel gating was observed prior to pressure ramp application were discarded to ensure that only initial channel gating events were measured. The relative gating pressures of each channel, $P_{X} / P_{\text {MscL }}$, where $X$ is either AtMSL1, A320V, or $E c$ MscS was calculated using the gating pressure at which the first sustained channel opening occurred ${ }^{36}$.
For relative gating pressure and conductance calculations, values from all possible traces for a given patch were averaged, then the average and standard deviations of all patch averages calculated. Patch averages of conductance and relative gating pressures were analyzed using one-way ANOVA followed by post hoc Tukey's tests to test for significant differences in relative gating pressure and conductance between AtMSL1, A320V, and $E c$ MscS.

To determine open-state lifetimes of AtMSL1 and A320V, the open dwell times of pressure-triggered channel openings were measured, with channels considered fully closed only when activity was lost for a period of $5 \mathrm{~s}$ or longer. Typically, only a single channel was observed in each patch; if multiple channels opened, only the first was used for analysis. Open dwell times observed for each channel were then binned into the following categories: 0-19.99 s, 20-39.99 s, 40-59.99 s, 60-79.99 s, $80+\mathrm{s}$, and the percentage of openings in each category calculated for AtMSL1 and A320V. Pipette potential was unclamped at $97.7 \mathrm{~s}$.

Reporting summary. Further information on research design is available in the Nature Research Reporting Summary linked to this article.

\section{Data availability}

Data supporting the findings of this manuscript are available from the corresponding author upon reasonable request. A reporting summary for this Article is available as a Supplementary Information file. Source data are provided with this paper. The cryo-EM maps have been deposited to Electron Microscopy Data Bank with accession codes EMD21444, EMD-21445, and EMD-21447. Atomic coordinates have been deposited to the Protein Data Bank (PDB) with accession codes: PDB 6VXM, PDB 6VXN, PDB 6VXP.

\section{Received: 22 March 2020; Accepted: 7 July 2020;}

Published online: 23 July 2020

\section{References}

1. Kung, C. A possible unifying principle for mechanosensation. Nature $\mathbf{4 3 6}$ 647-654 (2005).

2. Arnadóttir, J., Chalfie, M., Árnadóttir, J. \& Chalfie, M. Eukaryotic mechanosensitive channels. Annu. Rev. Biophys. 39, 111-137 (2010).

3. Hamilton, E. S., Schlegel, A. M. \& Haswell, E. S. United in diversity: mechanosensitive ion channels in plants. Annu. Rev. Plant Biol. 66, 113-137 (2015).

4. Sukharev, S. \& Corey, D. P. Mechanosensitive channels: multiplicity of families and gating paradigms. Sci. Signal. 2004, re4 (2004).

5. Katta, S., Krieg, M. \& Goodman, M. B. Feeling force: physical and physiological principles enabling sensory mechanotransduction. Annu. Rev. Cell Dev. Biol. 31, 347-371 (2015).

6. Ladoux, B. \& Mège, R. M. Mechanobiology of collective cell behaviours. Nat. Rev. Mol. Cell Biol. 18, 743-757 (2017).

7. Murthy, S. E., Dubin, A. E. \& Patapoutian, A. Piezos thrive under pressure: Mechanically activated ion channels in health and disease. Nat. Rev. Mol. Cell Biol. 18, 771-783 (2017).

8. Cox, C. D., Bavi, N. \& Martinac, B. Bacterial mechanosensors. Annu. Rev. Physiol. 80, 71-93 (2017).

9. Naismith, J. H. \& Booth, I. R. Bacterial mechanosensitive channels-MscS: evolution's solution to creating sensitivity in function. Annu. Rev. Biophys. 41, 157-177 (2012).

10. Basu, D. \& Haswell, E. S. Plant mechanosensitive ion channels: an ocean of possibilities. Curr. Opin. Plant Biol. 40, 43-48 (2017).

11. Peyronnet, R., Tran, D., Girault, T. \& Frachisse, J. M. Mechanosensitive channels: Feeling tension in a world under pressure. Front. Plant Sci. 5, 558 (2014).

12. Martinac, B., Buechner, M., Delcour, A. H., Adler, J. \& Kung, C. Pressuresensitive ion channel in Escherichia coli. Proc. Natl Acad. Sci. USA 84, 2297-2301 (1987).

13. Levina, N. et al. Protection of Escherichia coli cells against extreme turgor by activation of MscS and MscL mechanosensitive channels: identification of genes required for MscS activity. EMBO J. 18, 1730-1737 (1999).

14. Boer, M., Anishkin, A. \& Sukharev, S. Adaptive MscS gating in the osmotic permeability response in E. coli: the question of time. Biochemistry 50, 4087-4096 (2011).

15. Bass, R. B., Strop, P., Barclay, M. \& Rees, D. C. Crystal structure of Escherichia coli MscS, a voltage-modulated and mechanosensitive channel. Science 298, $1582-1587$ (2002).

16. Wang, W. et al. The structure of an open form of an E. coli mechanosensitive channel at $3.45 \AA$ resolution. Science 321, 1179-1183 (2008).

17. Zhang, X. et al. Structure and molecular mechanism of an anion-selective mechanosensitive channel of small conductance. Proc. Natl Acad. Sci. USA 109, 18180-18185 (2012). 
18. Pliotas, C. et al. The role of lipids in mechanosensation. Nat. Struct. Mol. Biol. 22, 991-998 (2015).

19. Rasmussen, T., Flegler, V. J., Rasmussen, A. \& Böttcher, B. Structure of the mechanosensitive channel MscS embedded in the membrane bilayer. J. Mol. Biol. 431, 3081-3090 (2019).

20. Reddy, B., Bavi, N., Lu, A., Park, Y. \& Perozo, E. Molecular basis of forcefrom-lipids gating in the mechanosensitive channel mscs. Elife https://doi.org/ 10.7554/elife.50486 (2019).

21. Lai, J. Y., Poon, Y. S., Kaiser, J. T. \& Rees, D. C. Open and shut: crystal structures of the dodecylmaltoside solubilized mechanosensitive channel of small conductance from Escherichia coli and Helicobacter pylori at $4.4 \AA$ and 4.1 Å resolutions. Protein Sci. 22, 502-509 (2013).

22. Steinbacher, S., Bass, R., Strop, P. \& Rees, D. C. Structures of the prokaryotic mechanosensitive channels MscL and MscS. Curr. Top. Membr. 58, 1-24 (2007).

23. Vásquez, V., Sotomayor, M., Cordero-Morales, J., Schulten, K. \& Perozo, E. A structural mechanism for MscS gating in lipid bilayers. Science 321, 1210-1214 (2008).

24. Akitake, B., Anishkin, A., Liu, N. \& Sukharev, S. Straightening and sequential buckling of the pore-lining helices define the gating cycle of MscS. Nat. Struct. Mol. Biol. 14, 1141-1149 (2007).

25. Malcolm, H. R., Heo, Y. Y., Elmore, D. E. \& Maurer, J. A. Defining the role of the tension sensor in the mechanosensitive channel of small conductance. Biophys. J. 101, 345-352 (2011).

26. Belyy, V., Anishkin, A., Kamaraju, K., Liu, N. \& Sukharev, S. The tensiontransmitting 'clutch' in the mechanosensitive channel MscS. Nat. Struct. Mol. Biol. 17, 451-458 (2010).

27. Veley, K. M. et al. Arabidopsis MSL10 has a regulated cell death signaling activity that is separable from its mechanosensitive ion channel activity. Plant Cell 26, 3115-3131 (2014).

28. Lee, C. P. et al. MSL1 is a mechanosensitive ion channel that dissipates mitochondrial membrane potential and maintains redox homeostasis in mitochondria during abiotic stress. Plant J. 88, 809-825 (2016).

29. Li, Y. et al. Structural insights into a plant mechanosensitive ion channel MSL1. Cell Rep. 30, 4518-4527.e3 (2020).

30. Denisov, I. G. \& Sligar, S. G. Nanodiscs for structural and functional studies of membrane proteins. Nat. Struct. Mol. Biol. 23, 481-486 (2016).

31. Böttcher, B., Prazak, V., Rasmussen, A., Black, S. S. \& Rasmussen, T. The structure of YnaI implies structural and mechanistic conservation in the MscS Family of mechanosensitive channels. Structure 23, 1705-1714 (2015).

32. Guo, Y. R. \& MacKinnon, R. Structure-based membrane dome mechanism for Piezo mechanosensitivity. Elife https://doi.org/10.7554/elife.33660 (2017).

33. Saotome, K. et al. Structure of the mechanically activated ion channel Piezol. Nature 554, 481-486 (2018).

34. Zhao, Q. et al. Structure and mechanogating mechanism of the Piezol channel. Nature 554, 487-492 (2018).

35. Anishkin, A. \& Sukharev, S. Water dynamics and dewetting transitions in the small mechanosensitive channel MscS. Biophys. J. 86, 2883-2895 (2004).

36. Edwards, M. D. et al. Pivotal role of the glycine-rich TM3 helix in gating the MscS mechanosensitive channel. Nat. Struct. Mol. Biol. 12, 113-119 (2005).

37. Haswell, E. S., Phillips, R. \& Rees, D. C. Mechanosensitive channels: what can they do and how do they do it? Structure 19, 1356-1369 (2011).

38. Lin, Y. C. et al. Force-induced conformational changes in PIEZO1. Nature 573, 230-234 (2019)

39. Zheng, S. Q. et al. MotionCor2: anisotropic correction of beam-induced motion for improved cryo-electron microscopy. Nat. Methods 14, 331-332 (2017).

40. Zhang, K. Gctf: real-time CTF determination and correction. J. Struct. Biol. 193, 1-12 (2016)

41. Zivanov, J. et al. New tools for automated high-resolution cryo-EM structure determination in RELION-3. Elife https://doi.org/10.7554/elife.42166 (2018).

42. Punjani, A., Rubinstein, J. L., Fleet, D. J. \& Brubaker, M. A. CryoSPARC: algorithms for rapid unsupervised cryo-EM structure determination. Nat. Methods 14, 290-296 (2017)

43. Waterhouse, A. et al. SWISS-MODEL: homology modelling of protein structures and complexes. Nucleic Acids Res. 46, W296-W303 (2018).

44. Pettersen, E. F. et al. UCSF Chimera-a visualization system for exploratory research and analysis. J. Comput. Chem. 25, 1605-1612 (2004).

45. Emsley, P., Lohkamp, B., Scott, W. G. \& Cowtan, K. Features and development of Coot. Acta Crystallogr. D. Biol. Crystallogr. 66, 486-501 (2010).

46. Adams, P. D. et al. PHENIX: a comprehensive Python-based system for macromolecular structure solution. Acta Crystallogr. D. Biol. Crystallogr. 66, 213-221 (2010).
47. Chen, V. B. et al. MolProbity: all-atom structure validation for macromolecular crystallography. Acta Crystallogr. D. Biol. Crystallogr. 66, 12-21 (2010)

48. Smart, O. S., Neduvelil, J. G., Wang, X., Wallace, B. A. \& Sansom, M. S. HOLE a program for the analysis of the pore dimensions of ion channel structural models. J. Mol. Graph. 14, 354-360, 376 (1996).

49. Eisenberg, D., Schwarz, E., Komaromy, M. \& Wall, R. Analysis of membrane and surface protein sequences with the hydrophobic moment plot. J. Mol. Biol. 179, 125-142 (1984).

50. Kumar, S., Stecher, G., Li, M., Knyaz, C. \& Tamura, K. MEGA X: molecular evolutionary genetics analysis across computing platforms. Mol. Biol. Evol. 35 1547-1549 (2018).

51. Edwards, M. D. et al. Characterization of three novel mechanosensitive channel activities in Escherichia coli. Channels 6, 272-281 (2012).

52. Martinac, B., Rohde, P. R., Cranfield, C. G. \& Nomura, T. Patch clamp electrophysiology for the study of bacterial ion channels in giant spheroplasts of E. coli. Methods Mol. Biol. 966, 367-380 (2013).

\section{Acknowledgements}

This work was supported by start-up funds from Washington University School of Medicine (to P.Y.) and an HHMI-Simons Faculty Scholar Grant to E.S.H. M.R and J.A.J. $\mathrm{F}$ are supported by the Washington University Center for Cellular Imaging, which is funded, in part by Washington University School of Medicine, the Children's Discovery Institute of Washington University and St. Louis Children's Hospital (CDI-CORE-2015505 and CDI-CORE-2019-813) and the Foundation for Barnes-Jewish Hospital (3770). A.M.S. is supported by funding from the Spencer T. and Ann W. Olin Fellowship for Women in Graduate Study and the William H. Danforth Plant Sciences Fellowship.

\section{Author contributions}

Z.D. and J.Z. performed biochemical preparations, cryo-EM experiments, structural determination and analysis. G.M. and A.M.S conducted electrophysiology experiments. M.R., J.A.J.F., and Z.D. performed cryo-EM data acquisition. P.Y. conceived and supervised the project. Z.D., G.M., A.M.S., E.S.H., and P.Y. analyzed the results and prepared the manuscript with input from all authors. Correspondence and requests for materials should be addressed to P.Y.

\section{Competing interests}

The authors declare no competing interests.

\section{Additional information}

Supplementary information is available for this paper at https://doi.org/10.1038/s41467 020-17538-1.

Correspondence and requests for materials should be addressed to P.Y.

Peer review information Nature Communications thanks Show-Ling Shyng and Sergei Sukharev for their contributions to the peer review of this work. Peer review reports are available.

Reprints and permission information is available at http://www.nature.com/reprints

Publisher's note Springer Nature remains neutral with regard to jurisdictional claims in published maps and institutional affiliations.

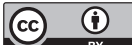

Open Access This article is licensed under a Creative Commons Attribution 4.0 International License, which permits use, sharing, adaptation, distribution and reproduction in any medium or format, as long as you give appropriate credit to the original author(s) and the source, provide a link to the Creative Commons license, and indicate if changes were made. The images or other third party material in this article are included in the article's Creative Commons license, unless indicated otherwise in a credit line to the material. If material is not included in the article's Creative Commons license and your intended use is not permitted by statutory regulation or exceeds the permitted use, you will need to obtain permission directly from the copyright holder. To view a copy of this license, visit http://creativecommons.org/ licenses/by/4.0/.

(C) The Author(s) 2020 